\title{
Chromosome Behaviour and Pachytene Morphology of Coix gigantea
}

\author{
Benny Jacob and J. Christopher \\ Department of Botany, University of Kerala, \\ Kariavattom, S. India
}

Acepted December 12, 1991

The genus Coix (tribe Maydeae, grass family) is characterised by its fruit-case or involucre, which is formed from the hardened floral leaf or bract. This genus consists of four species and $C$. gigantea often occurs as a pest in the paddy fields of South India (Bor 1960, Christopher and Jacob 1991). It is of great interest that this plant is having a life span of 3-4 months which is almost similar to that of paddy plants and appears to exhibit mimicry during the juvenile stages, as the leaves resemble paddy leaves. But at the flowering stage it reaches a height of about $1.5-3 \mathrm{~m}$, with profuse tillering and destroys the near-by paddy plants. The life cycle is completed along with paddy crop, dispersing the seeds in the paddy field for the next generation. Cytologically $C$. gigantea is well-known with three cyto-types $(2 n=10,2 n=20$ and $2 n=40$ ) on the basic number $X=5$ (Celarier 1957, Darlington and Wylie 1955, Peter Goldblatt 1984). Further Christopher and Jacob (1991) have reported a stabilized wild population of this species with $n=6$ and $2 n=12$ with perfectly regular meiosis and typical diploid behaviour. As Coix is characterised by the constant basic number $\mathrm{X}=5$, it is interesting to trace the origin of this cyto-type with $2 n=12$ and its relationship with the other species of the genus. The pachytene chromosome study offers additional evidence for establishing evolutionary relationships among plant groups (Mc Clintock 1929, Longley 1941, Rhoades 1955, Venkateswarlu and Reddi 1956, Venkateswarlu 1962, Mini et al. 1991 etc.). This is the first report presenting complete pachytene analysis of this cyto-type and an attempt has been made to elucidate the phylogenetic relationships of this species.

\section{Materials and methods}

Cytological observations were carried out on living plants collected in the field as well as those maintained in the Botanical garden, Kerala University. Seeds of Coix gigantea were collected from the paddy fields at Calicut (Kerala State) and Gobichettipalayam (Tamil $\mathrm{Nadu}$ ), germinated and maintained in the garden. Fifty plants were selected at random for cytological studies. Meiotic observations of pollen mother cells were made from young spikelets and mitotic studies from root tips fixed in 1:3 acetic alcohol fortified with ferric acetate and squashed in $2 \%$ acetocarmine. Prior to fixation root-tips were treated with $0.002 \%$ 8-hydroxyquinoline for $3 \mathrm{hr}$ at $0^{\circ} \mathrm{C}$ (Tjio and Levan 1950). Camera lucida drawings of pachytene chromosomes were made from temporary slides. Individual photographic prints of pachytene chromosomes were taken at the magnification of $\times 3000$ and explanatory diagrams were also prepared at the same magnification. The pachytene chromosomes were measured using a map-measurer and numbered I to VI according to the method of Mc Clintock (1929). The somatic chromosomes were classified according to the systems of Levan et al. (1964) and Stebbins (1971). The chromatin ratio is calculated as the ratio of total euchromatin length/ total heterochromatin length (Bahl and Tyagi 1988). Voucher specimens have been deposited in the Herbarium, Department of Botany, University of Kerala, Kariavattom. 


\section{Observations}

Coix gigantea Koenig ex Roxb. is an annual species having a life-span of about four months only. The plants are tall, robust, 1.5 to $3 \mathrm{~m}$ in height. The leaves are long and narrow, $50-80 \mathrm{~cm}$ in length and about $1.00-1.50 \mathrm{~cm}$ in breadth and resemble paddy leaves. The fruitcase is $0.4 \mathrm{~cm}$ in diameter hard-shelled, brownish, spheroid-elongated and shows a beak in the pre-mature seeds.

Somatic chromosome studies consistently showed 12 chromosomes in all the root-tip cells and ranged in length from 4.99 to $7.66 \mu \mathrm{m}$ (Fig. 1). The karyotype consists of four pairs of $\mathrm{m}$-type and two pairs of sm-type and of $2 \mathrm{~A}$ category. The first and fourth pair posses secondary constrictions in the long arm distal to the centromere. Meiosis showed the regular formation of six bivalents at diakinesis and metaphase $I$ in all the PMCs examined (Fig. 2). Two nucleoli of different sizes each associated with a separate bivalent were observed at diakinesis in about 90 per cent of the cells. Anaphase I segregation was also normal with six chromosomes at each pole. The pollen fertility was found to be about $85 \%$ and the plant propagates by seeds.

At the pachytene stage, the synapsis of homologous chromosomes was found to be complete and normal throughout the length of the bivalents. The pachytene chromosomes were of the differentiated type and quite large varying from 16.65 to $49.16 \mu \mathrm{m}$ in length. The centromere of each bivalent could be distinguished with accuracy and appeared as an unstained region flanked on either side by deeply staining regions. The individual bivalents could be identified on the basis of the heterochromatin flanking the centromere, position of centromere, arm ratio, presence of chromomeres, distinct terminal knobs etc. The characteristic morphological features of each bivalent are given below (Text Fig. 1, Table 1).

Table 1. Morphological details of the pachytene chromosomes Coix gigantea $(\mathrm{n}=6)$

\begin{tabular}{|c|c|c|c|c|c|c|}
\hline \multirow{2}{*}{$\begin{array}{l}\text { Chromosome } \\
\text { Number }\end{array}$} & \multicolumn{3}{|c|}{ Chromosome length in $\mu \mathrm{m}$} & \multirow{2}{*}{$\begin{array}{l}\text { Arm } \\
\text { ratio } \\
\mathrm{L} / \mathrm{S}\end{array}$} & \multirow{2}{*}{$\begin{array}{l}\text { Chromatin ratio } \\
\text { Euchromatin/ } \\
\text { Heterochromatin }\end{array}$} & \multirow{2}{*}{$\begin{array}{l}\text { Chromosome } \\
\text { type }\end{array}$} \\
\hline & Long arm & $\begin{array}{c}\text { Short arm } \\
\mathrm{S}\end{array}$ & Total Length & & & \\
\hline$I^{*}$ & $27.50 \pm 1.76$ & $21.66 \pm 0.98$ & $49.16 \pm 1.23$ & 1.26 & 1.28 & $\mathrm{~m}$ \\
\hline II & $22.82 \pm 2.17$ & $13.00 \pm 1.96$ & $35.82 \pm 2.06$ & 1.75 & 4.22 & $\mathrm{sm}$ \\
\hline III & $18.33 \pm 2.10$ & $16.82 \pm 1.47$ & $35.15 \pm 1.68$ & 1.08 & 1.29 & $\mathrm{~m}$ \\
\hline IV* & $10.83 \pm 0.96$ & $8.33 \pm 0.84$ & $19.16 \pm 0.87$ & 1.30 & 2.02 & $\mathrm{~m}$ \\
\hline $\mathrm{V}$ & $11.66 \pm 1.09$ & $5.83 \pm 0.62$ & $17.49 \pm 0.74$ & 2.00 & 0.98 & $\mathrm{sm}$ \\
\hline VI & $9.16 \pm 0.73$ & $7.49 \pm 1.64$ & $16.65 \pm 1.22$ & 1.22 & 1.86 & $\mathrm{~m}$ \\
\hline
\end{tabular}

* Nucleolar organizing chromosomes

\section{Chromosome I}

This is the longest of the complement (Figs. 3,3A) which is nucleolar organizing chromosome. Almost identical blocks of heterochromatin are found flanking the centromere on both arms. The presence of a significantly large distal block of heterochromatin on the short arm is the characteristic feature of this chromosome. Two macrochromomeres are also present on the short arm in between the two blocks of heterochromatin. The nucleolar organizing region is located towards the distal region of the long arm which terminates in a medium-sized knob. This chromosome is of m-type, the arm ratio is 1.26 and the chromatin ratio is 1.28 .

\section{Chromosome II}

This is the second largest of the complement, which is sub-median and the arm ratio is 1.75 (Figs. 4, 4A). The proximal heterochromatic regions are quite large and unequal in 
size. The chromatin ratio is quite high (4.22) and the heterochromatin is restricted to the proximal region only. The distal portions of both arms are entirely euchromatic.

\section{Chromosome III}

This median chromosome shows an arm ratio of 1.08 (Figs. 8, 8A). Almost equal amounts of heterochromatic blocks flank the centromere. Another block of heterochromatin is found in the middle of the long arm and two macrochromomeres towards its distal end. In the short arm one more heterochromatic block is found near the proximal heterochromatin. The chromatin ratio is 1.29 .

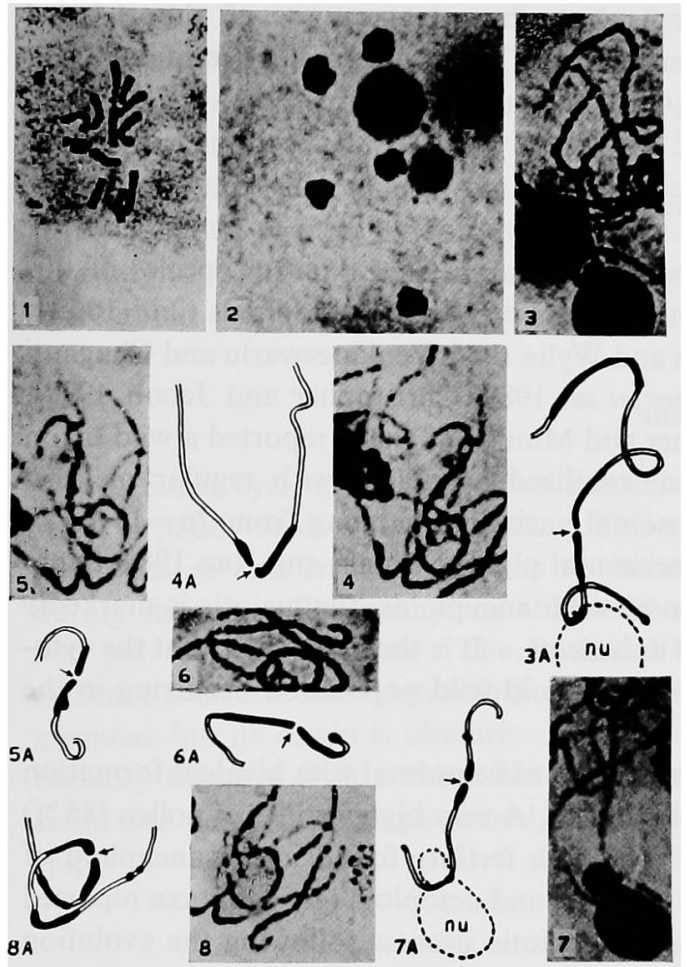

Figs. 1-8. 1, Coix gigantea. Somatic metaphase. $2 n=12(\times 835) 2$, Meiotic metaphase I. $n=6$ ( $\times 1200)$. 3-8. Pachytene chromosomes of $C$. gigantea $(\times 1430)$, Figs. $3-8$ are photomicrographs of pachytene chromosomes and Figs. $3 \mathrm{~A}$ to $8 \mathrm{~A}$ represent the corresponding explanatory diagrams. Arrows indicate the position of the centromere. $\mathrm{Nu}=$ Nucleolus.

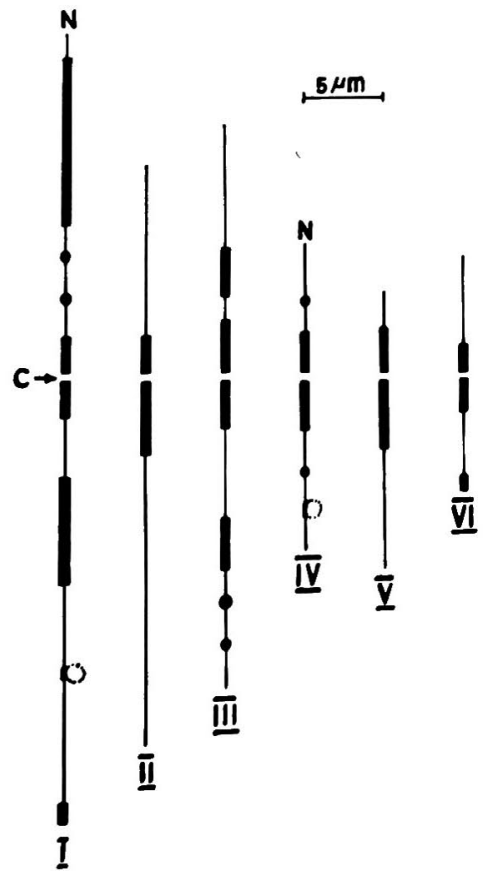

Text Fig. 1. Idiogram of the six pachytene bivalents of Coix gigantea. $\mathrm{C}=$ Centromere, $\mathrm{N}=$ Nucleolar organizing chromosomes.

\section{Chromosome IV}

This is the second pair of the complement with the nucleolar organizing region (NOR), located distally on the long arm (Figs. 7, 7A). This chromosome is almost 2.5 times smaller than the first nucleolar organizing pair with an arm ratio of 1.30. The centromere is flanked on both sides by almost equal amounts of heterochromatin. One macrochromomere each is also present on both arms towards the middle of the euchromatic region. The chromatin ratio is 2.02 , showing a significant decrease in the heterochromatin content than the first nucleolar organising pair. 


\section{Chromosome V}

This sub-median chromosome has an arm ratio 2.0 (Figs. 5, 5A). This is the only chromosome showing an overdominace in the heterochromatin content and the chromatin ratio is 0.98 . This chromosome is easily identifyable from the rest of the complement by the presence of a comparatively large block of proximal heterochromatin on each arm. About $2 / 3 \mathrm{rd}$ of the length of the short arm of this chromosome is heterochromatic while half of the long arm is heterochromatic.

\section{Chromosome VI}

This is the shortest of the complement, $16.65 \mu \mathrm{m}$ in length and arm ratio is 1.22 (Figs. 6 , 6A). The proximal heterochromatic blocks flanking the cetromere are of almost equal size and chromatin ratio is 1.86 . This is the second pair with a prominent terminal knob which is present in the long arm.

\section{Discussion}

A perusal of literature shows that the chromosome numbers of the four species of this genus reported so far form an euploid series ranging from diploids to octoploids $(2 n=10,20$, 30 and 40 ) on the base number $X=5$ (Darlington and Wylie 1955, Venkateswarlu and Chaganti 1973, Fedorov 1974, Goldblatt 1984, Christopher et al. 1989, Christopher and Jacob 1990). However Koul and Paliwal (1964) and Christopher and Mini (1988) have reported a wild taxon of Coix with $2 n=32$ chromosomes, which is a stabilized aneuploid with regular meiosis. Further unstable plants with aneuploid chromosomal variations ranging from $2 n=18$ to 22 have been reported to occur occasionally in experimental plots (Nirmala and Rao 1984, Sapre and Barve 1988, Barve and Sapre 1986). But in all such aneuploids, the meiosis is markedly disturbed with the formation of multivalents and univalents. It is threrfore clear that the cytotype with $2 \mathrm{n}=12$ now investigated is a stabilized aneuploid wild population occurring in the paddy fields of South India.

The meiosis in the plants with $2 \mathrm{n}=12$ has been found to be normal with bivalent formation and regular anaphase I segregation in most of the PMCs. A very high fertility of pollen (85\%) and normal seed set have also been observed. The high fertility found in the aneuploid of the present study as well as among the tetraploid $(2 n=20)$ and octoploid $(2 n=40)$ taxa reported earlier may have been the result of normalizing the meiotic process following the evolution of selectively advantageous genetic control that govern the process. This suggests evolution of some antiquity, where these taxa have had ample time to evolve genetic controls normalizing meiosis.

At the pachytene stage, there is complete pairing between all the homologous chromosomes and each of the six bivalents show certain distinguishing features, apart form the distribution of heterochromatin and arm ratio. The first bivalent is the longest of complement which is a nucleolar organizing chromosome and possess a terminal knob (Text Fig. 1). The IVth bivalent also shows a NOR towards the terminal end. A prominant terminal knob is exhibited by the VIth bivalent also. Venkateswarlu and Chaganti (1973) have recorded the presence of two NOC, in Coix aquatica with $2 n=10$. The present pachytene study has indicated, some degree of similarity between the IInd and Vth pairs, as they resemble each other in the distribution of proximal heterochromatin and arm ratio. Medium-sized terminal knobs are present in chromosomes I and VI. The knobs are considered to be very important in cytogenetical studies, where they are the cytological markers of chromosomes (Rhoades 1955). Knobs are useful to study the origin and distribution of species (Longley 1941, Stebbins 1971, Dennis and Peacock 1984). The presence of knobs in C. gigantea presently studied indicates 
its relationship with other cyto-types of this species which are also reported to have knobs.

As Coix is characterised by the basic number $X=5$, the cyto-type now studied, appears to be an aneuploid. But there is no clear evidence to establish its origin and especially the source of the two extra chromosomes found in this species. All the six bivalents show perfect pairing at pachytene and later stages of meiosis indicating their homologous nature. The pachytene analysis has shown a lot of similarities between the IInd and Vth pair. Evidently these two pairs appear as duplicated chromosmes. In chromosome $\mathrm{V}$ the chromatin ratio is 0.98 , which shows the presence of dominant heterochromatin when compared to euchromatic region. It is possible that some euchromatic regions of this bivalent have been lost during the course of evolution, of this stabilized aneuploid cyto-type. It is particularly interesting, this species with $2 n=12$ could be collected from South India only, especially as a weed in the paddy fields at a few places in Calicut (Kerala) and Gobichettypalayam (Tamil Nadu). Its robust nature with profuse tillering and strong root system destroys many neighbouring paddy plants and it completes its life-cycle within 3-4 months along with paddy. This species has survived to a certain extent from total eradication from paddy fields, up to this time, due to its resemblance to paddy plants at the juvenile stages (mimicry). A wide collection and detailed study of this cyto-type from different regions of South India might help us to understand more about the phylogenetic relationships of this species.

\section{Summary}

Detailed cytological studies have been made on Coix gigantea which often occurs as a weed in paddy fields of South India. This cyto-type shows the aneuploid chromosome numbers of $n=6$ and $2 n=12$. All the six bivalents are homologous showing complete pairing during meiosis. The pachytene studies show significant similarities between the IInd and Vth pair of chromosomes. The Ist and VIth pair show terminal knobs. The cytological evidence indicates the relationship of this cyto-type $(2 n=12)$ with the other cyto-types of $C$. gigantea, but its origin is obscure. This plant could survive in the paddy fields as it exhibits mimicry together with a short life-cycle. A wide collection fom different regions of South India and detailed cytological analysis might help us to understand its phylogenetic relationships.

\section{Acknowledgements}

The authors are thankful to the UGC for the financial assistance and to Dr. Susan Abraham, Head of the Department of Botany for facilities and encouragement.

\section{References}

Bahl, J. R. and Tyagi, B. R. 1988. Pachytene chromosomes of Coleus forskohlii. Curr. Sci. 57(6): 326-328. Barve, S. S. and Sapre, A. B. 1986. Mono-trisomic in Coix gigantea (Poaceae). Curr. Sci. 55(14): 660-661. Bor, N. L. 1960. Grasses of Burma, Ceylon, India and Pakistan. Pergamon Press, London.

Celarier, R. P. 1957. Cyto-taxonomy of Andropogoneae II. Cytologia 22: 160-183.

Christopher, J. and Benny Jacob. 1990. Cytology of a new Hexaploid cyto-type of Coix lacryma-jobi Linn. Cytologia 55: 57-60.

- and - 1991. Cytological studies of Coix gigantea Koenig ex Roxb. from South India. Cytologia 56: 265-268.

- and Mini, L.S. 1988. Karyomorphological studies of a new Taxon of Coix Linn. J. Soc. Cytol. and Genetics 23: 113-117.

-, - and Nagendra Pillai, T. 1989. Karyomorphological studies of Coix aquatica Roxb. Cytologia 54: 169-172. 
Darlington, C. D. and Wylie, A. P. 1955. Chromosome Atlas of Flowering Plants. G. Allen and Unwin, London. Dennis, E. S. and Peacock, W. J. 1984. Knob heterochromatin homology in maize and its relatives. J. Mol. Evol. 20: 341-350.

Fedorov, A. 1974. Chromosome Numbers of Flowering Plants. Rept. Koenigstein.

Goldblatt, Peter 1984. Index to Plant Chromosome Numbers. Missouri Botanical Garden.

Koul, A. K. and Paliwal, R. L. 1964. Morphology and cytology of a new species of Coix with 32 chromosomes Cytologia 29: 375-386.

Leavan, A., Fredga, K. and Sandberg, A. A. 1964. Nomenclature for centromeric position on chromos omes. Hereditas 52: 201-220.

Longley, A. E. 1941. Knob positions on teosinte chromosomes. J. Agric. Res. 62: 401-413.

Maguire, M. P. 1960. A study of pachytene chromosomes pairing in a Corn-Tripsacum hybrid derivatives. Genetics 45: 651-664.

Mc Clintock, B. 1929. Chromosome morphology of Zea mays. Science 69: 629-630.

Mini, L. S., Christopher, J. and Benny Jacob. 1991. Karyomorphological and phylogenetic studies on Hygroryza aristata (Retz.) Cytologia 56: 95-102.

Nirmala, A. and Rao. P. N. 1984. Chromosome instability in Coix gigantea. Can. J. Gene and Cytol. 26: 334-338.

Rhoades, M. M. 1955. Cytogenetics of Maize. In Corn and Corn Improvement. Ed. G. F. Sprague, Academic Press. New York.

Sapre, A. B. and Barve, S. S. 1988. Nullisomic-trisomic in Coix gigantea (Poaceae). Curr. Sci. 57: $90-91$. Stebbins, G. L. 1971. Chromosomal Evolution in Higher Plants. Addison-Wesley, London.

Tjio, J. H. and Levan, A. 1950. The use of oxyquinoline in chromosome analysis. Annal. Est. Exper. de. Avla dei $2: 21-64$.

Venkataswarlu, J. 1962. Some aspects of the study of pachytene chromosomes. Presidential address. Proc. 49th Indian Sci. Congr. Part II: 113-132.

- and Reddy V. R. 1956. Morphology of pachytene chromosomes and meiosis in Sorghum subglabrescens. J. Ind. Bot. Soc. 35: 344-356.

- and Chaganti, R. S. K. 1973. Job's Tears. Tech. Bull. No. 44. ICAR. New Delhi. 